\title{
Graph-Drawing Contest Report
}

\author{
Peter Eades ${ }^{1}$, Joe Marks ${ }^{2}$, and Stephen North ${ }^{3}$ \\ 1 Department of Computer Science \\ University of Newcastle \\ University Drive - Callaghan \\ NSW 2308, Australia \\ E-mail: eades@cs.newcastle.edu.au \\ ${ }^{2}$ MERL-A Mitsubishi Electric Research Laboratory \\ 201 Broadway \\ Cambridge, MA 02139, U.S.A. \\ E-mail: marks@merl.com \\ 3 AT\&T Research \\ 180 Park Ave., Bldg. 103 \\ Florham Park, N.J 07932-0971, U.S.A. \\ E-mail: north@research.att.com
}

\begin{abstract}
This report describes the Fourth Annual Graph Drawing Contest, held in conjunction with the 1997 Graph Drawing Symposium in Rome, Italy. The purpose of the contest is to monitor and challenge the current state of the art in graph-drawing technology $[2,3,4]$.
\end{abstract}

\section{Introduction}

Text descriptions of the three graphs for the 1997 contest can be found on the World Wide Web (WWW) at URL wwr. inf.uniroma3.it/calendar/gd97/contest/rules.html. Graph $A$ is an artificial graph that was designed as a special challenge for orthogonal graph-drawing algorithms. Graph B represents a collection of WWW pages and similarity measures between some of them. For both of these graphs an effective drawing had to communicate not only the edge connections between vertices, but also any vertex- or edge-attribute values peculiar to the graph. Graph $\mathrm{C}$ represents the calls made between a set of telephone numbers. Participants were required to submit a video of an interactive exploration through which they answered several specific questions about the graph.

Approximately 13 submissions were received by the contest deadline. The winners were selected by the organizers, and are shown below.

\section{Winning submissions}

\subsection{Graph A}

This directed graph contains 84 nodes and 333 edges. It was contrived without reference to a real-world application. The contest rules stated that only orthogonal drawings would be acceptable. 
This graph was designed to present a special challenge for standard techniques. Nevertheless, most contestants were able to produce excellent drawings of it. In fact, five of the six submissions were essentially the same ideal drawing. All five entries were jointly awarded first prize. The winning authors were:

- B. Bascary, B. Cattan, A. Cohen-Solal, M. Philip, and H. Szigeti, (szigeti@eurecom.fr), Telecom Paris, France.

- Vladimir Batagelj and Andrej Mrvar ([Vladimir.Batagelj, Andrej.Mrvar]@uni-lj.si), University of Ljubljana, Slovenia.

- Michael Kaufmann,(mk@informatik.uni-tuebingen.de), Universität Tübingen, Germany.

- Gunnar Klau, (guwek@mpi-sb.mpg.de), MPI für Informatik, Saarbrücken, Germany.

- Thomas Ziegler and Petra Mutzel, ([tziegler, mutzel]@mpi-sb.mpg.de), MPI für Informatik, Saarbrücken, Germany.

Figure 1 contains a representative drawing from the set of winners. Remarkably, the similar drawings seem to have been created using a variety of techniques. For example, Ziegler and Mutzel based their drawing on a planar embedding of a maximal subgraph [7]. The embedding was augmented by hand, and then edges were drawn using Tamassia's bend-minimization algorithm [8]. In contrast, Kaufmann used a spring embedder [6] and direct manipulation to unfold the original graph and uncover symmetries. He then used the "Kandinsky" approach [5], augmented with some ideas from [8], to draw the edges with a minimum number of bends.

\subsection{Graph B}

This graph contains 47 nodes and 264 edges. It represents a collection of WWW pages in which pages of similar content have been linked automatically. Each page is represented by a graph node with one attribute, the page title. Associated with each edge are the degree of similarity between the connected pages, and a list of words indicating the basis of commonality.

Few graph-drawing systems handle text labels well, and fewer still incorporate clustering or partitioning algorithms for grouping similar vertices. We therefore expected Graph B to be a tough challenge. Nevertheless, the winning drawing, shown in Figure 2, is an excellent visualization of the graph data. It was produced by Vladimir Batagelj and Andrej Mrvar ([Vladimir.Batagelj, Andrej.Mrvar]@uni-lj.si) from the University of Ljubljana, Slovenia, using the "Pajek" system (see http://vlado.fmf .uni-lj.si/pub/networks/pajek/). They used Ward's hierarchical clustering technique to compute vertex clusters [1], which were then rearranged automatically to minimize edge crossings. A final refinement of vertex locations was performed manually.

\subsection{Graph C}

This graph contains 452 nodes and 768 edges. It was extracted from a large telephone-call database. Graphs like this are used in fraud investigations. Asso- 


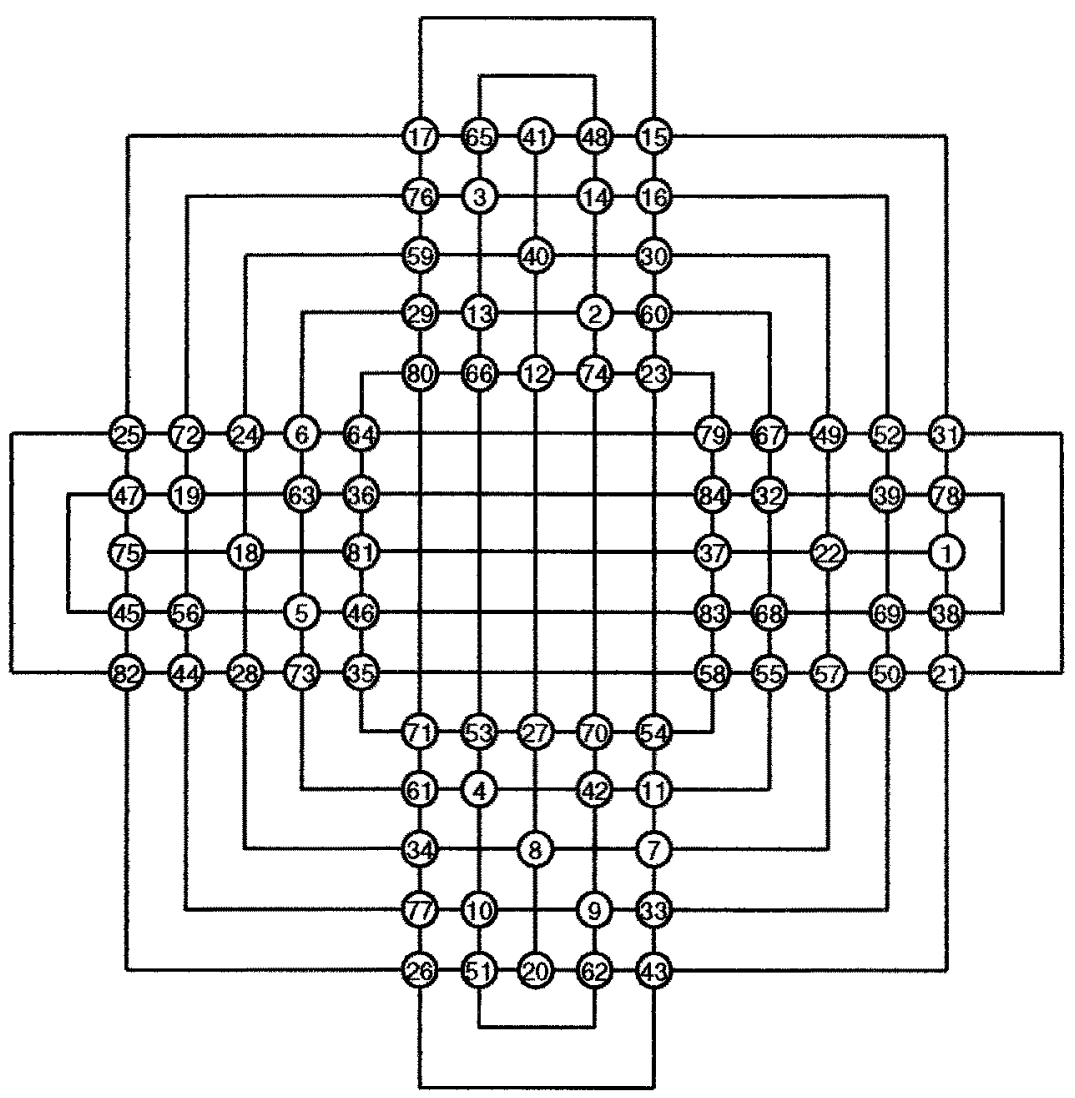

Fig. 1. Representative winner, Graph A.

ciated with the graph are several questions. Contestants were required to submit a video of their graph-drawing system being used to answer these questions.

There were two joint winners for this graph. The first video was submitted by Cristian Ghezzi (xtian@co.umist.ac.uk), of UMIST, Manchester, England. His Virtual Data Browser (VDB) is a 3D graph-drawing system (see http://ww. .co.umist.ac.uk/ xtian/VRML/vdb/vdb.html). It is written in Java, and uses SGML and VRML as the input and output file formats. A screen snapshot is shown in Figure $3 .{ }^{4}$ Much use is made of $3 \mathrm{D}$ navigation, color, and interactive exploration to answer the posed questions.

The other winning video was submitted by Falk Schreiber and Carsten Friedrich

${ }^{4}$ Of course, a screen snapshot does not do justice to the system, which is why video submissions were required in the first place. 


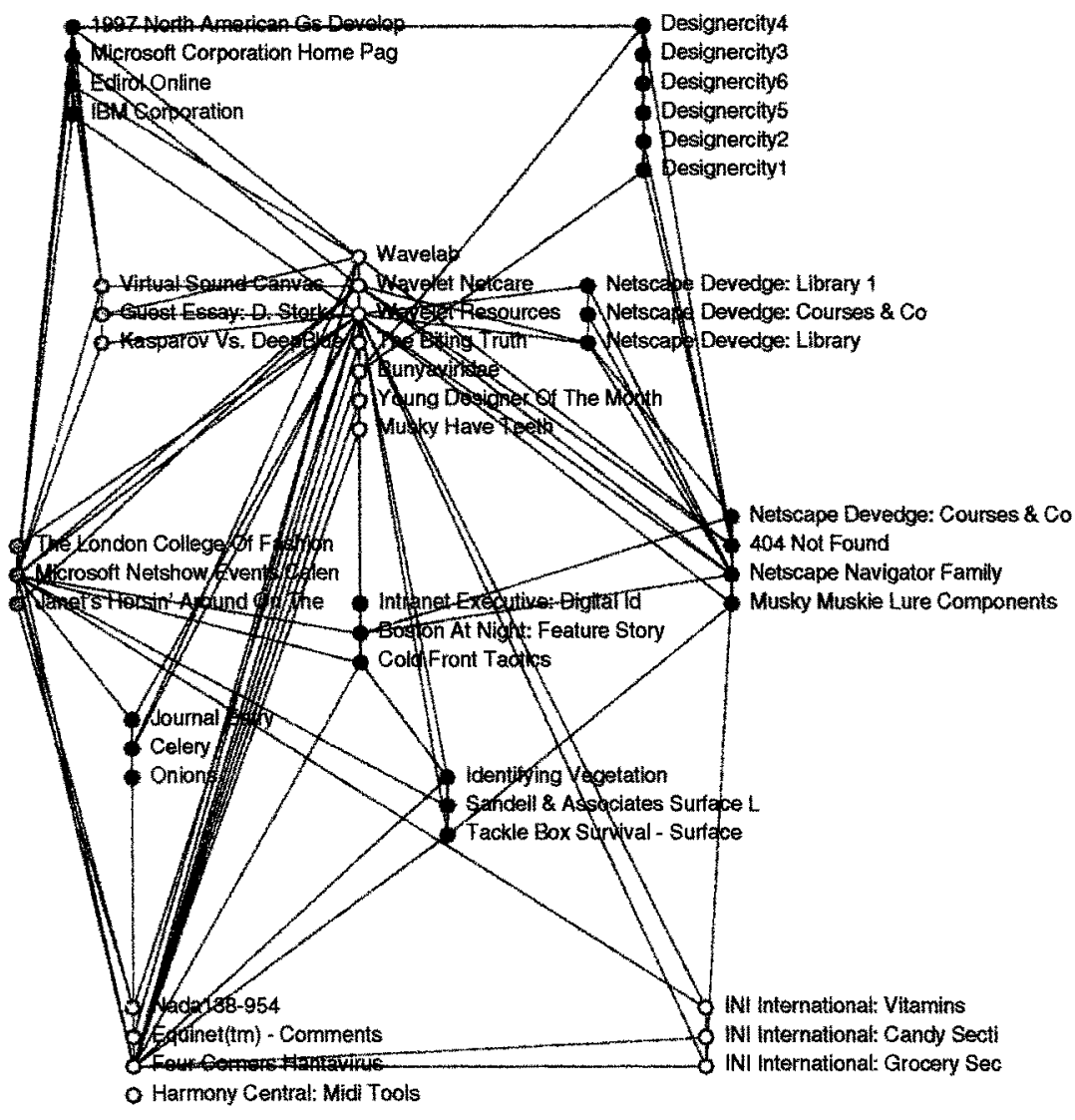

Fig. 2. Winner, Graph B.

([schreibe, friedric]@fmi.uni-passau.de) of Universität Passau, Germany. Their system is a more conventional 2D graph-drawing system, based on the "ffGraph" library (see http://www.fmi.uni-passau.de/friedric/ffgraph/ffgraph/ffgraph.html). A screen snapshot is shown in Figure 4. It shows clusters that arose naturally from a spring-method layout. These clusters were useful in answering several of the questions. Color and interactive exploration were also used to good effect.

\section{Observations and Conclusions}

The number of outstanding drawings for Graph A was a real surprise, especially because they were produced using several different techniques and ideas. In previous years, such a graph might not have been drawn well by most systems, 


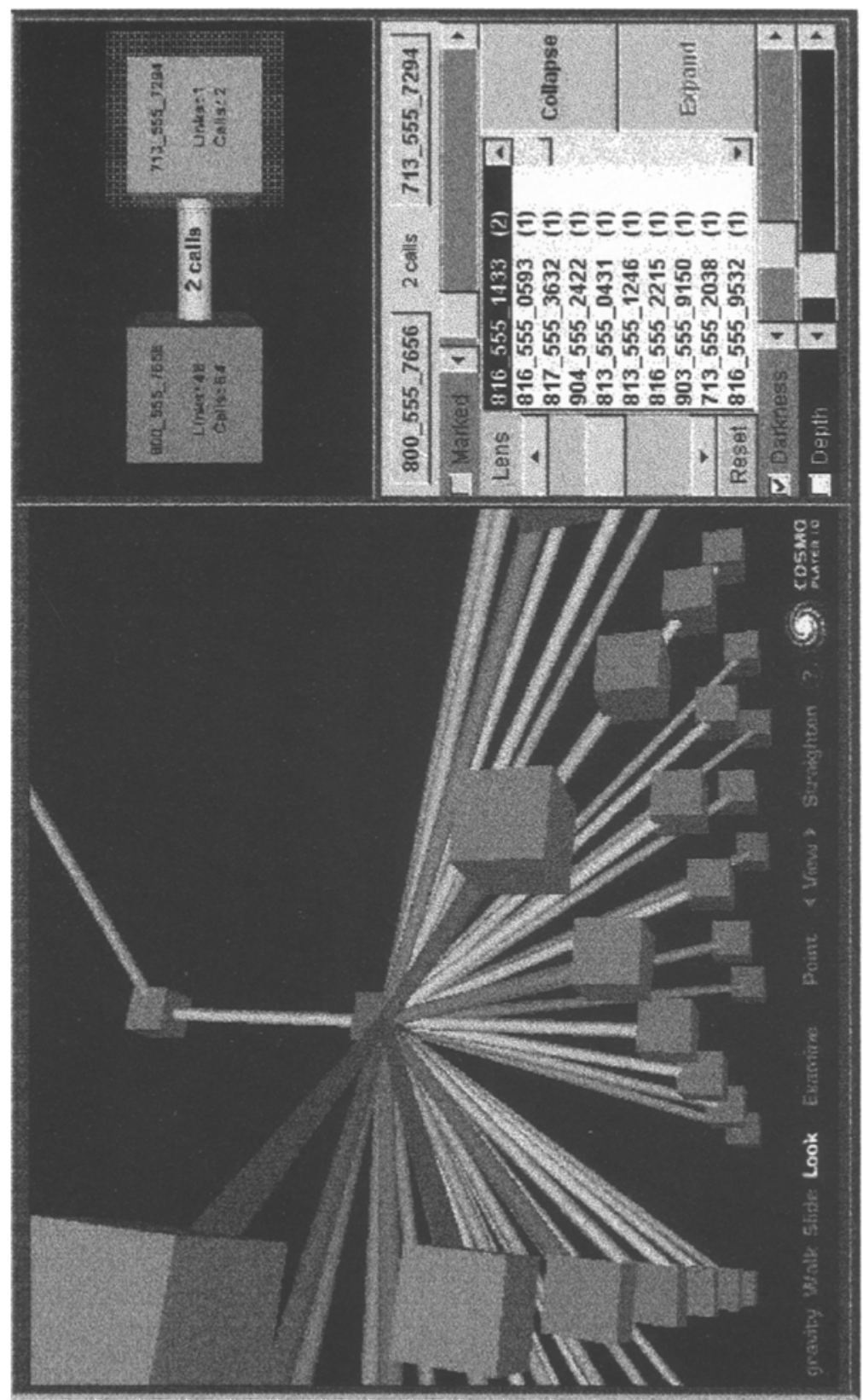

Fig. 3. Screen snapshot of joint winner, Graph C. 


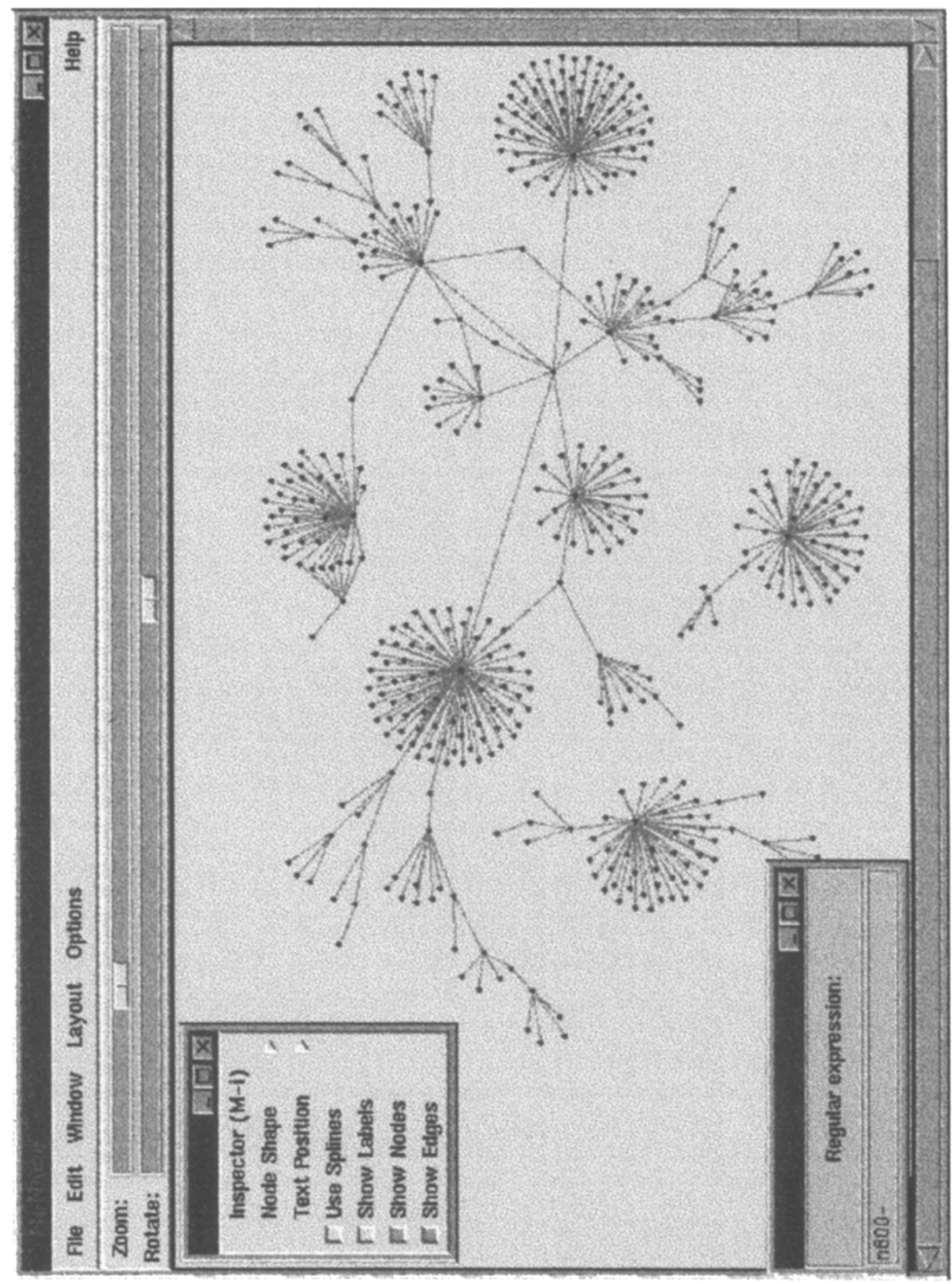

Fig. 4. Screen snapshot of joint winner, Graph C. 
but now it seems comfortably within range. This is encouraging evidence of the steady progress in graph drawing over the past few years.

It was also encouraging to receive entries for Graph $B$ that utilized partitioning and clustering ideas. These concepts have been underrepresented in previous contests, and yet have been studied since the very beginning of the field. Recent research on text-label placement does not yet appear to have been incorporated into many graph-drawing systems, but we expect this situation to change in the coming years. We intend to continue to include label placement as an element in future graph-drawing contests.

Soliciting video submissions was hopefully the start of a new trend. Although several groups attempted the production of videos for Graph $\mathrm{C}$, only two groups actually submitted ones. Hopefully they have blazed a trail for others to follow in the future. We continue to believe that a dynamic medium like video is essential for demonstrating interactive graph visualization, and incremental and $3 \mathrm{D}$ graph layout.

\section{Acknowledgments}

Sponsorship for this contest was provided by AT\&T Research, MERL-A Mitsubishi Electric Research Laboratory, and Lucent Technologies. Denny Bromley provided the data for Graph B.

\section{References}

1. M. Anderberg. Cluster Analysis for Applications. Academic Press, New York, 1973.

2. P. Eades and I. Marks. Graph-drawing contest report. In R. Tamassia and I. G. Tollis, editors, Lecture Notes in Computer Science: 894 (Proceedings of the DIMACS International Workshop on Graph Drawing '94), pages 143-146, Berlin Heidelberg, October 1994. Springer-Verlag.

3. P. Eades and J. Marks. Graph-drawing contest report. In F. J. Brandenburg, editor, Lecture Notes in Computer Science: 1027 (Proceedings of the Symposium on Graph Drawing GD '95), pages 224-233, Berlin Heidelberg, September 1995. Springer-Verlag.

4. P. Eades, J. Marks, and S. North. Graph-drawing contest report. In S. North, editor, Lecture Notes in Computer Science: 1190 (Proceedings of the Symposium on Graph Drawing GD '96), pages 129-138, Berlin Heidelberg, September 1996. Springer-Verlag.

5. U. Fößmeier and M. Kaufmann. Drawing high degree graphs with low bend numbers. In F. J. Brandenburg, editor, Lecture Notes in Computer Science: 1027 (Proceedings of the Symposium on Graph Drawing GD '95), pages 254-266, Berlin Heidelberg, September 1995. Springer-Verlag.

6. A. Frick, H. Mehldau, and A. Ludwig. A fast adaptive layout algorithm for undirected graphs. In R. Tamassia and I. G. Tollis, editors, Lecture Notes in Computer Science: 894 (Proceedings of the DIMACS International Workshop on Graph Drawing '94), pages 388-403, Berlin Heidelberg, October 1994. Springer-Verlag. 
7. M. Jünger and P. Mutzel. Solving the maximum weight planar subgraph problem by branch and cut. In G. Rinaldi and L. Wolsey, editors, Proceedings of the Third Conference on Integer Programming and Combinatorial Optimization (IPCO), pages 479-492, 1993.

8. R. Tamassia. On embedding a graph in the grid with the minimum number of bends. SIAM Journal on Computing, 16(3):421-444, 1987. 